\section{Correspondence on "Update on the diagnosis and management of systemic lupus erythematosus" by Fanouriakis et al}

This review ${ }^{1}$ provided a comprehensive update on recent developments in systemic lupus erythematosus (SLE) and proposed several new viewpoints. In terms of diagnosis, the authors were the first to put forward two types of clinical SLE, in addition to classified SLE. Particularly, in the European League Against Rheumatism (EULAR)/American College of Rheumatology score $<10$ category in figure 4 , the authors suggested that an immunological score of $\geq 2$ together with a clinical score of $\geq 6$ could be regarded as clinical SLE. Therefore, a patient with any joint involvement (six points) and positive antiphospholipid antibodies (two points) could be considered as having clinical SLE. However, in clinical practice, this patient could also be diagnosed with antiphospholipid syndrome. In addition, in the negative antinuclear antibody category, a patient with positive antiphospholipid antibodies and with joint (six points) and haematologic (four points) involvement could be diagnosed with clinical SLE under their proposed diagnostic approach. Clinically, a patient with lymphoma could also have these characteristics and the use of these diagnostic criteria might lead to misdiagnosis as SLE. Hence, we suggest that the criteria for clinical SLE, as defined in this review, require more careful consideration.

In terms of treatment, cyclophosphamide is also widely used in moderate activity SLE, especially in lupus nephritis (LN). This was not mentioned in figure 5 of this review. Furthermore, EULAR/European Renal Association-European Dialysis and Transplant Association guidelines suggest that the treatment of LN should be based on kidney biopsy results, ${ }^{2}$ which should be emphasised in the Lupus nephritis section of this review. In addition, rituximab could be an alternative treatment in refractory cases of LN.

Zhuochao Zhou, Yijun You, Fan Wang, Yue Sun, Jialin Teng ๑ , Honglei Liu, Xiaobing Cheng, Yutong Su $\odot$, Hui Shi $\odot$, Chengde Yang $\odot$, Junna Ye $\odot$

Department of Rheumatology and Immunology, Ruijin Hospital, Shanghai Jiao Tong University School of Medicine, Shanghai, China

Correspondence to Dr Junna Ye and Dr Chengde Yang, Department of Rheumatology and Immunology, Ruijin Hospital, Shanghai Jiao Tong University School of Medicine, Shanghai, China; yjn0912@qq.com, yangchengde@sina.com
Acknowledgements The authors thank Dr Yi Lu for her critical reading of this manuscript.

Contributors Study conception and manuscript writing: JY, CY, ZZ and YY. Manuscript revisions: JT, HL, XC, YS, HS and FW.

Funding This work was supported by the National Natural Science Foundation of China (81801592) and Clinical Research Plan of SHDC (SHDC2020CR4011).

Competing interests None declared.

Patient and public involvement Patients and/or the public were not involved in the design, or conduct, or reporting, or dissemination plans of this research.

Patient consent for publication Not required.

Ethics approval This study was approved by the Ethics Committee of Ruijin Hospital.

Provenance and peer review Not commissioned; internally peer reviewed.

(c) Author(s) (or their employer(s)) 2021. No commercial re-use. See rights and permissions. Published by BMJ.

$Z Z$ and $Y Y$ contributed equally.

$$
\text { Check for updates }
$$

To cite Zhou Z, You Y, Wang F, et al. Ann Rheum Dis Epub ahead of print: [please include Day Month Year]. doi:10.1136/annrheumdis-2021-220897

Received 31 May 2021

Accepted 10 July 2021

\section{SLinked}

http://dx.doi.org/10.1136/annrheumdis-2021-221151

Ann Rheum Dis 2021;0:1. doi:10.1136/annrheumdis-2021-220897

ORCID iDs

Jialin Teng http://orcid.org/0000-0003-1016-9064

Yutong Su http://orcid.org/0000-0003-0488-2939

Hui Shi http://orcid.org/0000-0002-3574-8807

Chengde Yang http://orcid.org/0000-0002-3720-634X

Junna Ye http://orcid.org/0000-0002-2823-4349

\section{REFERENCES}

1 Fanouriakis A, Tziolos N, Bertsias G, et al. Update on the diagnosis and management of systemic lupus erythematosus. Ann Rheum Dis 2021;80:14-25.

2 Fanouriakis A, Kostopoulou M, Cheema K, et al. 2019 update of the joint European League against rheumatism and European renal Association-European dialysis and transplant association (EULAR/ERA-EDTA) recommendations for the management of lupus nephritis. Ann Rheum Dis 2020;79:713-23. 